\title{
Representing preorders with injective monotones
}

\author{
Pedro Hack ${ }^{1}$ (D) Daniel A. Braun ${ }^{1} \cdot$ Sebastian Gottwald ${ }^{1}$
}

Accepted: 13 December 2021 / Published online: 24 January 2022

(C) The Author(s) 2022

\begin{abstract}
We introduce a new class of real-valued monotones in preordered spaces, injective monotones. We show that the class of preorders for which they exist lies in between the class of preorders with strict monotones and preorders with countable multiutilities, improving upon the known classification of preordered spaces through realvalued monotones. We extend several well-known results for strict monotones (Richter-Peleg functions) to injective monotones, we provide a construction of injective monotones from countable multi-utilities, and relate injective monotones to classic results concerning Debreu denseness and order separability. Along the way, we connect our results to Shannon entropy and the uncertainty preorder, obtaining new insights into how they are related. In particular, we show how injective monotones can be used to generalize some appealing properties of Jaynes' maximum entropy principle, which is considered a basis for statistical inference and serves as a justification for many regularization techniques that appear throughout machine learning and decision theory.
\end{abstract}

Keywords Multi-utility representation - Richter-Peleg function · Majorization · Uncertainty preorder · Maximum entropy

\section{Introduction}

The set of all preordered spaces $(X, \preceq)$ is structured according to how well their preorder can be represented by real-valued monotones, that is, functions $u: X \rightarrow \mathbb{R}$ such that $x \preceq y$ implies $f(x) \leq f(y) \forall x, y \in X$ (Evren \& Ok, 2011; Ok, 2002). Two

Pedro Hack

pedro.hack@uni-ulm.de

Daniel A. Braun

daniel.braun@uni-ulm.de

Sebastian Gottwald

sebastian.gottwald@uni-ulm.de

1 Institute of Neural Information Processing, Ulm University, 89081 Ulm, Germany 
major classification methods can be distinguished depending on whether one considers a single monotone (Alcantud et al., 2016) or a whole family $U$ of monotones encapsulating all the information in $\preceq$, called a multi-utility (Evren \& Ok, 2011). More precisely, if $U$ is a multi-utility for $(X, \preceq)$ then $\forall x, y \in X$ we have $x \preceq y$ if and only if $u(x) \leq u(y) \forall u \in U$. Without further constraints, monotones and multi-utilities are, however, not very useful from a classification perspective as they exist for any preordered space. They become more useful when adding constraints. For example, there are preordered spaces without strict monotones, that is, without monotones $u$ such that, $u(x)<u(y)$ whenever $x \prec y$. ${ }^{1}$ Strict monotones, also known as Richter-Peleg functions, have been extensively studied (Alcantud et al., 2013, 2016; Peleg, 1970; Richter, 1966) and are related to other features of the preorder such as its maximal elements. In the case of multi-utilities, the cardinality is an important property for the classification of preordered spaces, with countable multi-utilities playing a central role (Bevilacqua et al., 2018c). Of particular importance are utility functions (Debreu, 1954, 1964), that is, multi-utilities consisting of a single function. ${ }^{2}$

Here, we introduce injective monotones, which are monotones $u$ such that $u(x)=$ $u(y)$ implies both $x \preceq y$ and $y \preceq x$. Preorders for which they exist form a category between preorders with strict monotones and preorders with countable multiutilities, as we show in Propositions 1, 5 and 8. Hence, we improve on the existing classification of preorders by adding a new distinct class. More precisely, in Sect. 3, we define injective monotones and prove some simple properties. After discussing their relation to optimization in Sect. 4, we take a look at the role of multi-utilities in Sect. 5, in particular, we construct injective monotones from countable multiutilities and show that the converse does not hold. Finally, in Sect. 6, we consider separability properties of preorders that are sufficient for the existence of strict and injective monotones, introducing a new notion of Debreu separability, that allows to extend previous results on strict monotones to corresponding analogues for injective monotones.

In the following section, we introduce our running example to which we come back several times throughout the development of the general theory. In particular, we discuss the relation between the uncertainty preorder from majorization theory (Arnold, 2018), which has Shannon entropy as a strict monotone, and the maximum entropy principle that appears in many different parts of science.

\section{Example: the uncertainty preorder and Shannon entropy}

The outcome of a random variable with a narrow probability distribution is easier to predict than the outcome of a random variable with a less concentrated distribution. For example, the result of throwing an unbalanced coin is easier to predict than the one of a balanced coin. In other words, a wider distribution contains more uncertainty than a narrower distribution. This idea is captured by a binary relation

\footnotetext{
${ }^{1}$ Here, $x \prec y$ means $x \preceq y$ and $\neg(y \preceq x)$.

2 Notice, having a utility function implies $\preceq$ is total, that is, any pair of points $x, y \in X$ can be related by $\preceq$.
} 
on the space $\mathbb{P}_{\Omega}$ of probability distributions on a set $\Omega$ : the uncertainty preorder $\preceq_{U}$, defined for finite $\Omega$ by

$$
p \preceq_{U} q \Longleftrightarrow u_{i}(p) \leq u_{i}(q) \quad \forall i \in\{1, . .,|\Omega|-1\}
$$

where $u_{i}(p):=-\sum_{n=1}^{i} p_{n}^{\downarrow}$ and $p^{\downarrow}$ denotes the decreasing rearrangement of $p$ (same components as $p$ but ordered decreasingly). Notice, $\preceq_{U}$ is known in mathematics, economics, and quantum physics as majorization (Arnold, 2018; Brandao et al., 2015; Hardy et al., 1952; Marshall et al., 1979), originally developed by Lorenz (1905) and Dalton (1920) among others, to measure wealth and income inequality. An intuitive way to think of $p \preceq_{U} q$ is that $q$ is the result of finitely many transfers of pieces of probability from a more likely to a less likely option in $p$ (Gottwald \& Braun, 2019). In other words, $q$ is more spread out or less biased, and thus, contains more uncertainty than $p$. For instance, a Dirac distribution is the smallest, and the uniform distribution is the largest, with respect to $\preceq_{U}$, among all distributions on $\Omega$.

There is, however, a downside to this intuitive notion of uncertainty: what if $p$ and $q$ do not have this relationship? For example, if $p=(0.6,0.2,0.2,0, . ., 0)$ and $q=(0.5,0.4,0.1,0, . ., 0)$, then $p$ and $q$ cannot be related by $\preceq_{U}$. Instead, the most common way to measure uncertainty is to use an entropy functional, such as the Shannon entropy, $H(p):=-\mathbb{E}_{p}[\log p]$, or one of various alternative entropy proposals, including Renyi entropy (Rényi et al., 1961), Tsallis entropy (Tsallis, 1988), and many more (Csiszár, 2008). Even though, in general, $\preceq_{U}$ cannot be fully represented by any of these so-called generalized entropies $F$, it is noteworthy that all of them are monotones with respect to $\preceq_{U}{ }^{3}$ While the converse is not true for any single $F$, there are collections $\mathcal{F}$ which constitute a multi-utility, e.g., in the case of finite $\Omega, \mathcal{F}=\left\{\sum_{n=1}^{|\Omega|} f\left(p_{n}\right) \mid f\right.$ concave $\}$ (Schur, 1923), or even $\mathcal{F}=\left\{u_{i}\right\}_{i=1}^{|\Omega|-1}$ by the definition of $\preceq_{U}$ (1).

The preference towards unbiased distributions, that is represented by any monotone of $\preceq_{U}$, is of particular relevance in the maximum entropy principle, where (Shannon) entropy serves as a counter-acting force against the bias towards the maximal elements of a given "energy" function E. Going back to the principle of insufficient reason (Bernoulli, 1713), today the maximum entropy principle appears in virtually all branches of science. For example, it is often used as a general principle to explain the raison d'etre behind all kinds of "soft" versions of known machine learning methods, especially in reinforcement learning (Fox et al., 2016; Williams \& Peng, 1991), but also in models of robust and resource-aware decision making (Maccheroni et al., 2006; Ortega \& Braun, 2013; Still, 2009; Tishby \& Polani, 2011). Basically, whenever there appears a trade-off between precision and uncertainty, there is a good chance that the maximum entropy principle is applied (Gottwald \& Braun, 2020).

The underlying goal of the maximum entropy principle is to select a typical distribution among a set of candidate distributions satisfying a given constraint, usually of the form $\langle E\rangle_{p}=c$, where $\langle E\rangle_{p}$ denotes the expectation of a random variable $E$ with respect to the probability measure $p$. In Wallis' derivation of the

\footnotetext{
3 Since $\preceq_{U}$ is not total for any $|\Omega|>2$, it has no utility function
} 
maximum entropy principle, typicality is measured by the number of possibilities of assigning $n$ elements among $N$ groups, under the limit of infinitely many elements $(n \rightarrow \infty)$ such that the statistical probabilities $p_{i}=\frac{n_{i}}{N}$ of belonging to a specific group $i$ remain finite ( $n_{i}$ denotes the number of elements in group $i$ ) Jaynes (2003). However, we can also think of typicality as containing the least amount of bias, or in other words, the maximal amount of uncertainty. Thus, when considering the uncertainty preorder $\preceq_{U}$ as the most basic way to decide about the difference in uncertainty between two distributions, then the ultimate goal of the maximum entropy principle becomes to obtain the maximal elements of $\preceq_{U}$, inside the given constraint set.

Even though, generally, we are not guaranteed to find all maximal elements of $\preceq_{U}$ when maximizing entropy, maximum entropy solutions are in fact maximal elements of $\preceq_{U}$, as entropy is a strict monotone. Furthermore, since the maximum entropy principle maximizes a strictly concave functional $H$ over a convex subset, it yields a unique maximal element of $\preceq_{U}$. In contrast, injective monotones, which exist for $\preceq_{U}$ (see Proposition 5), preserve this uniqueness property up to equivalence (see Proposition 3), without asking for the additional structural requirements of concavity.

\section{Injective monotones}

A preorder $\preceq$ on a set $X$ is a reflexive $(x \preceq x \forall x \in X$ ) and transitive ( $x \preceq y$ and $y \preceq z$ implies $x \preceq z \forall x, y, z \in X)$ binary relation. A tuple $(X, \preceq)$ is called a preordered space. An antisymmetric ( $x \preceq y$ and $y \preceq x$ imply $x=y \forall x, y \in X$ ) preorder $\preceq$ is called a partial order. The relation $x \sim y$, defined by $x \preceq y$ and $y \preceq x$, forms an equivalence relation on $X$, that is, it fulfills the reflexive, transitive and symmetric ( $x \sim y$ if and only if $y \sim x \forall x, y \in X$ ) properties. Notice, a preorder $\preceq$ is a partial order on the quotient set $X / \sim=\{[x] \mid x \in X\}$, consisting of all equivalence classes $[x]=\{y \in X \mid y \sim x\}$. In case $x \preceq y$ and $\neg(x \sim y)$ for some $x, y \in X$ we say $y$ is strictly preferred to $x$, denoted by $x \prec y$. If $\neg(x \preceq y)$ and $\neg(y \preceq x)$, we say $x$ and $y$ are incomparable, denoted by $x \bowtie y$. Whenever there are no incomparable elements, a preordered space is called total. By the Szpilrajn extension theorem (Szpilrajn, 1930), every partial order can be extended to a total order, that is, to a partial order that is total. Notice, the set $\mathbb{P}_{\Omega}$ of probability distributions on $\Omega$ equipped with the uncertainty preorder $\preceq_{U}$ forms a non-antisymmetric preordered space, because equivalent elements are only equal up to permutations (Arnold, 2018).

A real-valued function $f: X \rightarrow \mathbb{R}$ is called a monotone if $x \preceq y$ implies $f(x) \leq f(y)$. If also the converse is true, then $f$ is called a utility function. Furthermore, if $f$ is a monotone and $x \prec y$ implies $f(x)<f(y)$, then $f$ is called a strict monotone (or a Richter-Peleg function Alcantud et al., 2016).

Definition 1 (Injective monotones) A monotone $f: X \rightarrow \mathbb{R}$ on a preordered space $(X, \preceq)$ is called an injective monotone if $f(x)=f(y)$ implies $x \sim y$, that is, if $f$ is injective considered as a function on the quotient set $X / \sim$. 
Clearly, an injective monotone is also a strict monotone, since $x \prec y$ and $f(x)=$ $f(y)$ contradicts injectivity. The converse is not true, for example, Shannon entropy is a strict monotone for the uncertainty preorder $\preceq_{U}$ (Appendix A.1) but not an injective monotone, nor a utility. In fact, preorders that have an injective monotone form a class in between preorders that have a strict monotone and preorders that have a utility function.

\section{Proposition 1}

(i) There are preorders with strict monotones but without injective monotones.

(ii) There are preorders with injective monotones and without utility functions.

\section{Proof}

(i) Consider $(\mathcal{P}(\mathbb{R}), \preceq)$, the power set $\mathcal{P}(\mathbb{R})$ of the reals equipped with the preorder $\preceq$ defined by $U \preceq V$ if and only if $U=V$, or $U=\{0\}$ and $V=\{1\}$. Then $v: \mathcal{P}(\mathbb{R}) \rightarrow \mathbb{R}$, given by $v(\{1\})=1$ and $v(U)=0 \forall U \neq\{1\}$, is a strict monotone. However, there cannot be injective monotones, because here $|\mathcal{P}(\mathbb{R}) / \sim|=|\mathcal{P}(\mathbb{R})|$ and by Cantor's theorem the cardinality of $\mathbb{R}$ is strictly smaller than the cardinality of $\mathcal{P}(\mathbb{R})$.

(ii) Consider $(\mathbb{R}, \preceq)$, where $x \preceq y$ if and only if $x \leq y$ and $x, y \neq 0$ or $x=y=0$. The identity $I: \mathbb{R} \rightarrow \mathbb{R}$ is an injective monotone. However, $(\mathbb{R}, \preceq)$ is nontotal since $0 \bowtie x \forall x \in \mathbb{R} /\{0\}$ and, thus, has no utility function.

Since every preorder has a monotone (constant functions) and there are preorders without strict monotones (see Appendix A.2.1 for an example), we arrive at the picture shown in Fig. 1. Notice, the closer we are to the center, the better a monotone represents the underlying preorder. In particular, injective monotones contain more information about the preorder than strict monotones.

Nevertheless, in well-behaved cases, it is possible to construct an injective monotone out of a strict monotone. A negative example is the strict monotone that appears in the proof of $(i)$ in Proposition 1, which maps uncountably many incomparable elements to a single number (zero). If a strict monotone fails to be an injective monotone because of only countably many points, however, then it can easily be turned into an injective monotone by consecutive elimination.

Proposition 2 A preordered space $(X, \preceq)$ has an injective monotone if and only if it has a strict monotone $f$ whose non-injective set

$$
I_{f}:=\{x \in X \mid \exists y \in X \text { s.t. } f(x)=f(y) \text { and } x \bowtie y\}
$$

is countable.

Proof By definition, for an injective monotone $f$, we have $I_{f}=\emptyset$. Conversely, consider a strict monotone $f$ with a countable non-injective set. Given a numeration $\left\{x_{n}\right\}_{n \geq 0}$ of $I_{f}$, define $f_{0}: X \rightarrow \mathbb{R}$ by 


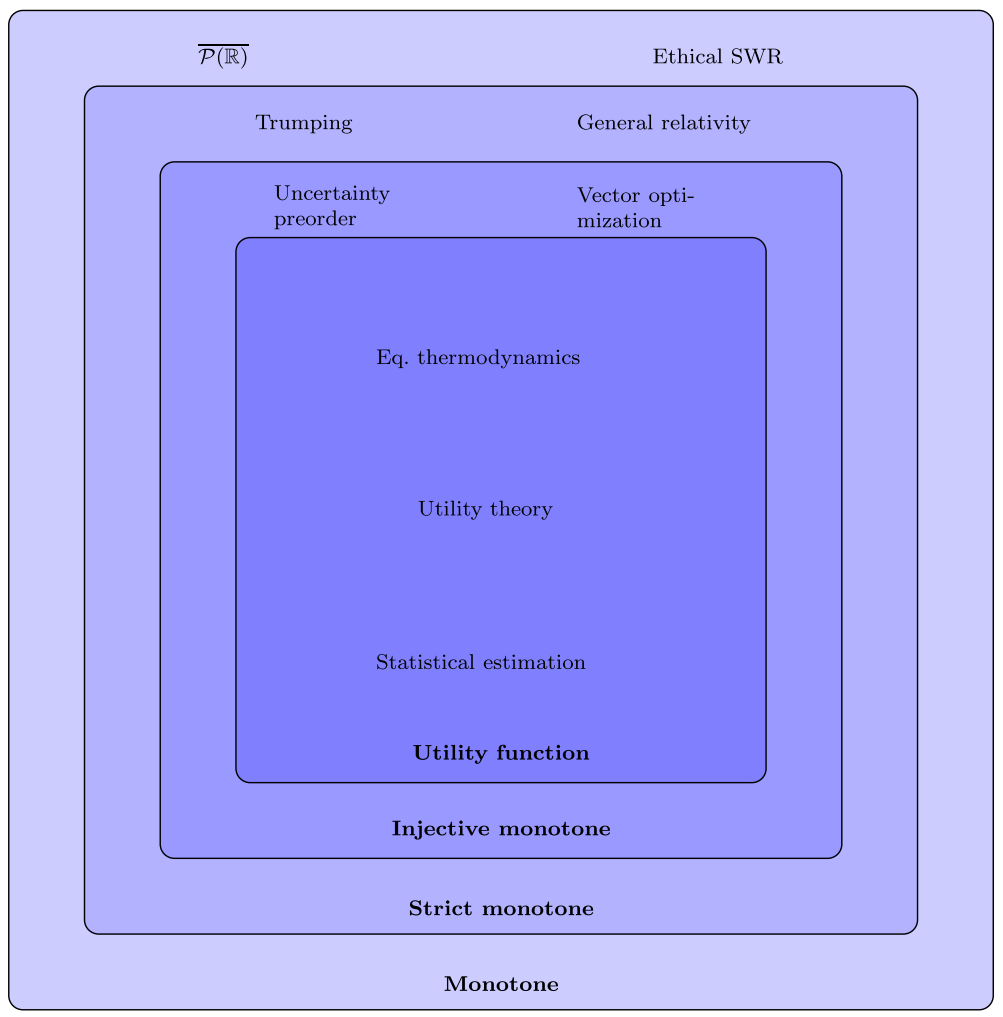

Fig. 1 Classification of preordered spaces according to the existence of various classes of monotones. The newly introduced class of preorders with injective monotones is strictly contained within the class of preorders with strict monotones, is strictly larger than the class of preorders with utility functions, and contains commonly used examples such as the uncertainty preorder and vector optimization. See Sect. 7 and Appendix A.2.1 for a short description of the other examples in the figure. Notice we have classified both trumping and general relativity according to current knowledge, they may have injective monotones (see Sect. 7)

$$
f_{0}(x):= \begin{cases}f(x)+1 & \text { if } f(x) \geq f\left(x_{0}\right) \text { and } \neg\left(x \sim x_{0}\right) \\ f(x) & \text { else. }\end{cases}
$$

Notice, by definition, $\forall x, y \in X, f(x) \leq f(y)$ implies $f_{0}(x) \leq f_{0}(y), I_{f_{0}} \subset I_{f}$, and $f_{0}$ is injective up to equivalence at $x_{0}$, in particular $x_{0} \notin I_{f_{0}}$. Therefore, we can consecutively eliminate the elements in $I_{f}$ by defining for all $n \in \mathbb{N}, f_{n}(x):=f_{n-1}(x)+2^{-n}$ if $f_{n-1}(x) \geq f_{n-1}\left(x_{n}\right)$ and $\neg\left(x \sim x_{n}\right)$, and $f_{n}(x):=f_{n-1}(x)$ otherwise, analogously to $f_{0}$. It is then straightforward to see that the pointwise limit $c(x):=\lim _{n \rightarrow \infty} f_{n}(x)$ exists for all $x \in X$ and that $c$ is an injective monotone.

Notice, the technique in the proof of Proposition 2 does not work if $I_{f}$ is uncountable. In particular, it cannot be used to construct an injective monotone from Shannon entropy $f=H$ for the uncertainty preorder $\preceq_{U}$, because if $N:=|\Omega| \geq 3$ then for all $c \in(0, \log N)$ there are $p, q \in \mathbb{P}_{\Omega}$ with $c=H(p)=H(q)$ but $p \bowtie q$ (see 
Appendix A.1). In other words, we can construct an injective map $g:(0, \log N) \rightarrow$ $I_{H}$ and thus $I_{H}$ has the same cardinality as $\mathbb{R}$, in particular $I_{H}$ is not countable.

\section{Relating monotones to optimization}

An element $x \in X$ is called a maximal element of $\preceq$ if there exists no $y \in X$ such that $x \prec y$. For any $B \subseteq X$, an element $x \in B$ is called a maximal element of $\preceq$ in $B$ if there exists no $y \in B$ such that $x \prec y$.

Definition 2 (Representing maximal elements) We say, a function $f: X \rightarrow \mathbb{R}$ is effective for $B \subseteq X$ if $\operatorname{argmax}_{B} f \neq \emptyset$, where $\operatorname{argmax}_{B} f:=\{x \in B \mid \nexists y \in$ $B$ such that $f(x)<f(y)\}$. We say, a function $f: X \rightarrow \mathbb{R}$ represents maximal elements of $\preceq$, if for any $B \subseteq X$

$$
\operatorname{argmax}_{B} f \subseteq B_{\bar{M}}^{\prec},
$$

where $B_{\bar{M}}$ denotes the set of maximal elements of $\preceq$ in $B$. Similarly, we say, a function $f$ injectively represents maximal elements of $\preceq$, if for any $B \subseteq X$ for which $f$ is effective, there exists $x_{0} \in B_{\bar{M}}^{\prec}$ such that

$$
\operatorname{argmax}_{B} f=\left.\left[x_{0}\right]\right|_{B}
$$

where $\left.\left[x_{0}\right]\right|_{B}$ is the equivalence class of $x_{0}$ restricted to $B$. Moreover, we say, $(X, \preceq)$ has an (injective) optimization principle if there exists a function $f: X \rightarrow \mathbb{R}$ which (injectively) represents maximal elements of $\preceq$.

Even though Shannon entropy does not represent $\preceq_{U}$ as a utility, its property as a strict monotone guarantees that its maxima are in fact maximal elements of $\preceq_{U}$, i.e., $H$ represents maximal elements of the uncertainty preorder according to Definition 2. Indeed, any $p \in \operatorname{argmax}_{B} H$ is a maximal element of $\preceq_{U}$ for any $B \subseteq$ $\mathbb{P}_{\Omega}$ on which $H$ is effective, as $p \prec q$ for some $q \in B$ would lead to the contradiction $H(q)>H(p)$. In fact, representing maximal elements is closely related to being a monotone for preorders in general.

Proposition 3 Given a preordered space $(X, \preceq)$ and a monotone $u: X \rightarrow \mathbb{R}$, then

(i) $\quad u$ is a strict monotone if and only if $u$ represents maximal elements of $\preceq$.

(ii) $\quad u$ is an injective monotone if and only if $u$ injectively represents maximal elements of $\preceq$.

\section{Proof}

(i) If $u$ is a strict monotone, then $\operatorname{argmax}_{B} u \subseteq B \preceq$ (by the same argument as for entropy). Conversely, consider $x, y \in X$ with $x \prec y$. For $B:=\{x, y\}$, we have $B_{\bar{M}}^{\prec}=\{y\}$ and thus $\{y\}=\operatorname{argmax}_{B} u$, i.e., $u(x)<u(y)$. 
(ii) For any $B \subseteq X$ on which $u$ is effective, if $x, y \in \operatorname{argmax}_{B} u$, we have $u(x)=$ $u(y)$ and, since $u$ is an injective monotone, $x \sim y$. Conversely, consider $x, y \in$ $X \quad$ and $B:=\{x, y\}$. If $u(x)=u(y)$ then by hypothesis $\{x, y\}=$ $\operatorname{argmax}_{x \in B}\{u(x)\}=\left.\left[x_{0}\right]\right|_{B}$ for some $x_{0} \in B$. In particular, $x \sim y$.

Notice, for the "if" part in (ii), we do not have to assume that $u$ is a monotone, that is, if the maxima of some real-valued function $u$ form an equivalence class in the set of maximal elements, then it already follows that $u$ is a monotone.

For any preordered space $(X, \preceq)$, thus, the existence of a strict monotone implies the existence of an optimization principle and the existence of an injective monotone is equivalent to the existence of an injective optimization principle. One can contrast the global injective representation of maximal elements which characterizes injective monotones in Proposition 3 with local approaches, for some specific $B \subseteq X$, present in the literature (Bevilacqua et al., 2018b; White, 1980).

Choosing a particular strict monotone $u$ and optimizing it in a set $B$ might, however, not yield all the maximal elements in $B_{\bar{M}}^{\prec}$. For example, take $p, q \in \mathbb{P}_{\Omega}$ with $p \bowtie q$ and $H(p)<H(q)$, then $B=\{p, q\}$ has the two maximal elements $p$ and $q$, but $\operatorname{argmax}_{B} H=\{p\}$. Notice, this is not only an issue for trivial examples like this, but also happens for the maximum entropy principle with linear constraint sets. In particular, if $B=\{p \mid\langle E\rangle=c\}$, for a given random variable $E$ and some $c \in \mathbb{R}$, crosses two incomparable elements that turn out to be maximal (see Fig. 2), then only part of the actual maximal elements of $\preceq_{U}$ can be found by maximizing entropy.

Similarly, while optimizing an injective monotone in a set $B$ results in equivalent elements, in general, we only find a slice of the set of all maximal elements in $B$. In fact, for every maximal element $x$ in $B_{\bar{M}}^{\prec}$, we can construct an injective monotone $c$ such that $x \in \operatorname{argmax} c$ (e.g., in the proof of Proposition 4 below, take $c_{x}$ if $x \in A_{c}$ and $c$ otherwise). This means that the problem of selecting a maximal equivalence class can be replaced by the problem of selecting an injective monotone.

In the following section, we show that injective monotones exist for a large class of preorders, including the uncertainty preorder.

\section{Relating monotones to multi-utilities}

Although it is not possible to capture all information about a non-total preorder using a single real-valued function, a family of functions may be used instead. A family $V$ of real-valued functions $v: X \rightarrow \mathbb{R}$ is called a multi-utility (representation) of $\preceq$ if

$$
x \preceq y \Longleftrightarrow v(x) \leq v(y) \forall v \in V .
$$

Whenever a multi-utility consists of strict monotones, it is called a strict monotone (or Richter-Peleg Alcantud et al., 2016) multi-utility (representation) of $\preceq$. 


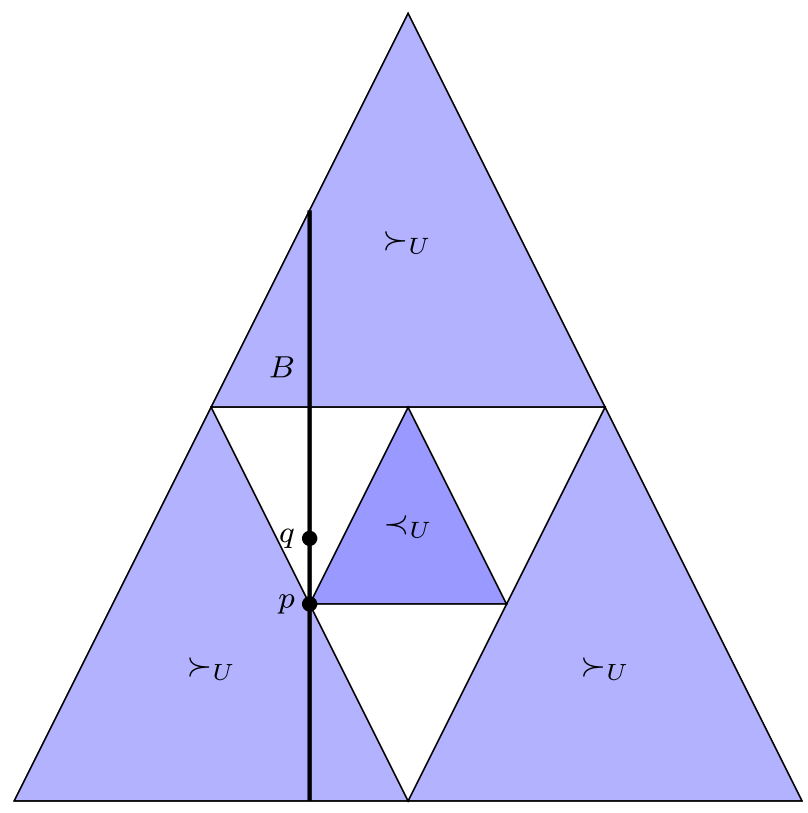

Fig. 2 Example for when the maximum entropy principle does not yield all maximal elements of $\preceq_{U}$ in some $B \subseteq P_{\Omega}$. Here, we show the usual visualization of the 2-simplex, that is, the set of all probability distributions in $P_{\Omega}$ for $|\Omega|=3$. Let the energy function $E$ be given by $E\left(x_{1}\right):=1, E\left(x_{2}\right):=-1$, and $E\left(x_{3}\right):=0$, and let $B$ be given by the constraint $\langle E\rangle=\frac{1}{4}$, represented by the vertical line. The distribution $p=(1 / 2,1 / 4,1 / 4)$ is a maximal element in $B$, because any other element of $B$ is either smaller than $p$ (belongs to an outer blue region) or incomparable (belongs to the white region). However, $q=$ $(9 / 20,4 / 20,7 / 20)$ is in $B$ and $H(p)<H(q)$. As a result, $p$ is a maximal element of $B$ which is not obtained via the maximum entropy principle

Analogously, if the multi-utility consists of injective monotones, we call it an injective monotone multi-utility (representation) of $\preceq$.

It is straightforward to see that every preordered space $(X, \preceq)$ has the multiutility $\left(\chi_{i(x)}\right)_{x \in X}$, where $\chi_{A}$ denotes the characteristic function of a set $A$ and $i(x):=\{y \in X \mid x \preceq y\}$ (Ok, 2002). Moreover, if there exists a strict monotone $u$, then a multi-utility $U$ only consisting of strict monotones can easily be constructed from a given multi-utility $V$ by $U:=\{v+\alpha u\}_{v \in V, \alpha>0}$ (Alcantud et al., 2013). Even though this construction does not work directly in the case of injective monotones, a simple modification does, where special care is given to incomparable elements.

Proposition 4 Let $(X, \preceq)$ be a preordered space. There exists an injective monotone if and only if there exists an injective monotone multi-utility.

Proof Consider w.l.o.g. an injective monotone $c: X \rightarrow(0,1)$ and

$$
A_{c}:=\{x \in X \mid \exists y \in X \text { s.t. } x \bowtie y, c(x)<c(y)\},
$$

i.e., the part of $X$ that has incomparable elements $y$ with strictly larger values of $c$. For all $x \in A_{c}$, let $c_{x}:=c+\chi_{i(x)}$. Notice, by construction $c_{x}(y)=c(y)<1 \leq c_{x}(x)$ for 
all $y \in X$ with $x \bowtie y$. Using $c(X) \subseteq(0,1)$ and the fact that $c$ is an injective monotone, it is straightforward to see that $C:=\{c\} \cup\left\{c_{x}\right\}_{x \in A_{c}}$ is an injective monotone multi-utility.

Note that the injective monotone multi-utility in the proof of Proposition 4 can be chosen to have cardinality of at most $\mathfrak{c}$, the cardinality of the continuum, because it is enough to have one $c_{x}$ per equivalence class $[x] \in X / \sim$, and, whenever an injective monotone exists, $|X / \sim| \leq \mathfrak{c}$.

The cardinality of multi-utilities plays an important role. In particular, special interest lies in preordered spaces with countable multi-utilities. In practice, countable multi-utilities are often used to define preordered spaces. For example, the uncertainty preorder $\preceq_{U}$ is defined in (1) by a countable (finite) multi-utility. Also, many applications in multicriteria optimization (Bevilacqua et al., 2018b; Ehrgott 2005 ) rely on preordered spaces defined by countable multi-utilities. It turns out that for the existence of strict monotones, such as entropy for $\preceq_{U}$, it is sufficient to have a countable multi-utility (Alcantud et al., 2016, Section 4). Here, we show that countable multi-utilities actually imply the existence of injective monotones, which, due to Proposition 1, improves upon (Alcantud et al., 2016).

Proposition 5 If, for a given preordered space $(X, \preceq)$, there exists a countable multi-utility, then there exists an injective monotone.

This means that the class of preordered spaces where countable multi-utilities exist is contained in the class of preordered spaces where an injective monotone exists (cf. Fig. 1). However, there exist preordered spaces with injective monotones, i.e., by Proposition 4, with injective monotone multi-utilities of cardinality $\mathfrak{c}$, but without countable multi-utilities (see Proposition 8).

For the uncertainty preorder $\preceq_{U}$, which is defined in (1) through a finite multiutility, Proposition 5, therefore, guarantees the existence of injective monotones. Moreover, we can see a possible construction in (3) below.

By a slight adaptation of the proof of Proposition 5, we obtain the stronger.

Proposition 6 For a given preordered space $(X, \preceq)$, there exists a countable multiutility if and only if there exists a countable multi-utility only consisting of injective monotones.

This improves upon (Alcantud et al., 2016, Proposition 4.1), where it is shown that a countable multi-utility exists if and only if a countable strict monotone multiutility exists. Notice, however, while for the proof in Alcantud et al. (2016), one can simply modify each member of a given multi-utility separately_-similarly as we did for the construction in Proposition 4-our proof of Proposition 6 relies on a more indirect technique, where each member of the resulting injective monotone multiutility does not have a direct relationship to a non-injective member of the given multi-utility.

For the proofs of Propositions 5 and 6, we rely on the following basic facts, the proofs of which can be found in the appendix. 
Lemma 1 Let $X$ be a set. Given $r \in\left(0, \frac{1}{2}\right)$ and a countable family $\left(A_{n}\right)_{n \geq 0}$ of subsets $A_{n} \subseteq X$, define the function $c: X \rightarrow \mathbb{R}$ by

$$
c(x):=\sum_{n \geq 0} r^{n} \chi_{A_{n}}(x) .
$$

Then, $c(x)<c(y)$ if and only if, for the first $m \in \mathbb{N}$ with $\chi_{A_{m}}(x) \neq \chi_{A_{m}}(y)$, we have $\chi_{A_{m}}(x)<\chi_{A_{m}}(y)$.

The following characterizations of injective monotones and countable multiutilities follow by straightforward manipulations of their definitions.

Lemma 2 Let $(X, \preceq)$ be a preordered space. A monotone $u$ is an injective monotone if and only if

$$
x \prec y \Rightarrow u(x)<u(y) \text { and } x \bowtie y \Rightarrow u(x) \neq u(y) .
$$

A collection $U$ of monotones is a multi-utility if and only if

$$
\neg(y \preceq x) \Rightarrow \exists u \in U \text { s.t. } u(x)<u(y) .
$$

A subset $A \subseteq X$ of a preordered space $(X, \preceq)$ is called decreasing if for all $x \in A$, $y \preceq x$ implies $y \in A$. Analogously, a subset $A \subseteq X$ is called increasing, if for all $x \in A, x \preceq y$ implies that $y \in A$ (Mehta, 1986a). We say a family $\left(A_{n}\right)_{n \in \mathbb{N}}$ of subsets $A_{n} \subseteq X$ separates $x$ from $y$, if there exists $n \in \mathbb{N}$ with $x \notin A_{n}$ and $y \in A_{n}$.

Lemma 3 Let $\left(A_{n}\right)_{n \geq 0}$ be a family of increasing sets.

(i) If, for all $x, y \in X$ with $x \prec y,\left(A_{n}\right)_{n \geq 0}$ separates $x$ from $y$, then the function $c: X \rightarrow \mathbb{R}$ defined in (3) is a strict monotone for all $r \in(0,1)$.

(ii) If in addition, for all $x, y \in X$ with $x \bowtie y,\left(A_{n}\right)_{n \geq 0}$ separates $x$ from $y$, or $y$ from $\mathrm{x}$, then $c$ is an injective monotone for all $r \in\left(0, \frac{1}{2}\right)$.

Notice, the construction of strict and injective monotones in Lemma 3 is based on Lemma 1 and is analogous to constructions that appear in the literature, where one typically uses a value of $r=\frac{1}{2}$ (e.g., Alcantud et al., 2016; Mehta, 1977; Ok, 2002). The requirement of $r<\frac{1}{2}$ in Lemmas 1 and 3 ensures that the resulting monotone is injective. In fact, as can be seen from the proof of Lemma 1 in the appendix, for $r \in(0,1)$ we have $r^{m}=\frac{r}{1-r} \sum_{n>m} r^{n}$. A value of $r \in\left(0, \frac{1}{2}\right)$ thus enables the strict estimate $r^{m}>\sum_{n>m} r^{n}$, which is exactly where the injectivity up to equivalence of $c$ in Lemma 3 rests.

Proof of Proposition 5 For a countable multi-utility $\left(u_{m}\right)_{m \in M}$ and $q \in \mathbb{Q}$, consider the increasing sets $A_{m, q}:=u_{m}^{-1}([q, \infty))$. It suffices to show that $\left(A_{n}\right)_{n \geq 0}$, where $A_{n}:=A_{m_{n}, q_{n}}$ for some enumeration $n \longmapsto\left(m_{n}, q_{n}\right)$ of $M \times \mathbb{Q}$, satisfies $(i)$ and (ii) in Lemma 3. If $x \prec y$ or $x \bowtie y$, then, by (5), in both cases there exists $m \in M$ with 
$u_{m}(x)<u_{m}(y)$. Hence, we can choose $q \in \mathbb{Q}$ with $u_{m}(x)<q<u_{m}(y)$, in particular, $x \notin A_{m, q}$ and $y \in A_{m, q}$.

Proof of Proposition 6 Let $\left(u_{m}\right)_{m \in M}$ be a countable multi-utility and let $c$ be an injective monotone of the form (3) constructed from the increasing sets $A_{n}$ in the proof of Proposition 5. We define, for any pair $(m, p) \in \mathbb{N}$ such that $m<p, \varphi_{m, p}$ : $\mathbb{N} \rightarrow \mathbb{N}$ which permutes $m$ and $p$ without changing any other natural number. For each $\varphi_{m, p}$, we define an injective monotone $c_{m, p}$ of the form (3) constructed from $\left(A_{\varphi_{m, p}(n)}\right)_{n \geq 0}$, the increasing sets used to define $c$ reordered by $\varphi_{m, p}$. Since $\{c\} \cup$ $\left\{c_{m, p}\right\}_{(m, p) \in \mathbb{N}^{2}, \mathrm{~m}<\mathrm{p}}$ is composed of injective monotones, it suffices to show (5) holds to conclude there exists a countable multi-utility composed of injective monotones. Consider, thus, $x, y \in X$ such that $\neg(y \preceq x)$. If $x \prec y$, then $c(x)<c(y)$ by definition. Assume now $x \bowtie y$. If $c(x)<c(y)$, then we have finished. Otherwise, we have $x \in A_{m}$ and $y \notin A_{m}$ for the first $m \in \mathbb{N}$ such that $\chi_{A_{m}}(x) \neq \chi_{A_{m}}(y)$ by Lemma 1. Since there exists some $p \in \mathbb{N} p>m$ such that $y \in A_{p}$ and $x \notin A_{p}$, the first $n \in \mathbb{N}$ such that $\chi_{A_{\varphi_{m, p}}(n)}(x) \neq \chi_{A_{\varphi_{m, p}(n)}}(y)$ is $n=m$. We conclude $c_{m, p}(x)<c_{m, p}(y)$ by Lemma 1 , since we have $\chi_{A_{\varphi_{m, p}}(m)}(x)=\chi_{A_{p}}(x)<\chi_{A_{p}}(y)=\chi_{A_{\varphi_{m, p}(m)}}(y)$.

Countable separating families such as the ones in Lemma 3 have been used to characterize preordered spaces with continuous utility functions (Herden, 1989), generalizing theorems of Peleg and Mehta (1981). In a similar spirit, Alcantud et al. (2013) extend a result by Bosi and Zuanon (2013) about upper semicontinuous multi-utilities based on separating families, showing that there exists a countable multi-utility if and only if there exists a countable family of decreasing subsets that $\forall x, y \in X$ with $\neg(y \preceq x)$ separates $x$ from $y$ (Alcantud et al., 2013, Proposition 2.13). Using Lemma 3 and the characterizations in Lemma 2, we immediately get the following analogous characterizations for preorders with strict and injective monotones, the proofs of which can be found in Appendix A.2.4.

Proposition 7 Let $(X, \preceq)$ be a preordered space.

(i) There exists a strict monotone if and only if there exists a countable family of increasing subsets that $\forall x, y \in X$ with $x \prec y$ separates $x$ from $y$.

(ii) There exists an injective monotone if and only if there exists a countable family of increasing subsets that satisfies (i) and (ii) in Lemma 3.

Countable separating families are a useful tool to improve the classification of preordered spaces by monotones. In particular, we use them in Proposition 8 to show the converse of Proposition 5 is false, that is, there are preordered spaces where injective monotones exist and countable multi-utilities do not.

Proposition 8 There are preordered spaces with injective monotones and without countable multi-utilities.

Proof Consider $X:=[0,1] \cup[2,3]$ equipped with $\preceq$ where 


$$
x \preceq y \Longleftrightarrow\left\{\begin{array}{l}
x=y \\
x \in[0,1], y \in[2,3] \text { and } y \neq x+2
\end{array}\right.
$$

$\forall x, y \in X$ (see Fig. 3 for a representation of $\preceq$ ). Notice $(X, \preceq)$ is a preordered space and the identity map $i_{d}: X \rightarrow \mathbb{R}$ is an injective monotone. We will show any family $\left(A_{i}\right)_{i \in I}$, where $A_{i} \subseteq X$ is increasing $\forall i \in I$ and $\forall x, y \in X$ such that $\neg(y \preceq x)$ there exists some $i \in I$ such that $x \notin A_{i}$ and $y \in A_{i}$, is uncountable. Since the existence of some $\left(A_{i}\right)_{i \in I}$ with those properties and countable $I$ is equivalent to the existence of a countable multi-utility (Alcantud et al., 2013, Proposition 2.13), we will get there is no countable multi-utility for $X$. Consider a family $\left(A_{i}\right)_{i \in I}$ with the properties above and, for each $x \in[0,1], y_{x}:=x+2$. Since $x \bowtie y_{x}$ by definition, there exists some $A_{x} \in\left(A_{i}\right)_{i \in I}$ such that $x \in A_{x}$ and $y_{x} \notin A_{x}$. We fix such an $A_{x}$ for each $x \in[0,1]$ and consider the map $f:[0,1] \rightarrow\left(A_{i}\right)_{i \in I}, x \mapsto A_{x}$. Given $x, z \in[0,1] x \neq z$, if we assume $z \in A_{x}$, then, since $A_{x}$ is increasing and $z \prec y_{x}$ as $y_{x} \neq z+2$, we would have $y_{x} \in A_{x}$, a contradiction. Notice, analogously, we get a contradiction if we assume $x \in A_{z}$ and, therefore, $A_{x} \neq A_{z}$. Thus, $A_{x}=A_{z}$ implies $x=z$ and we have, by injectivity of $f,|[0,1]| \leq\left|\left(A_{i}\right)_{i \in I}\right|$. As a consequence, $X$ has no countable multi-utility.

As we have seen in this section, the concept of separating families is closely related to the existence of monotones. In particular, this link is apparent when considering sets of the form $u^{-1}([q, \infty))$ for some monotone $u$ and $q \in \mathbb{Q}$, allowing to translate the two concepts into each other (see the proofs of Propositions 5 and 6). There is another rich class of separability properties of preordered spaces providing

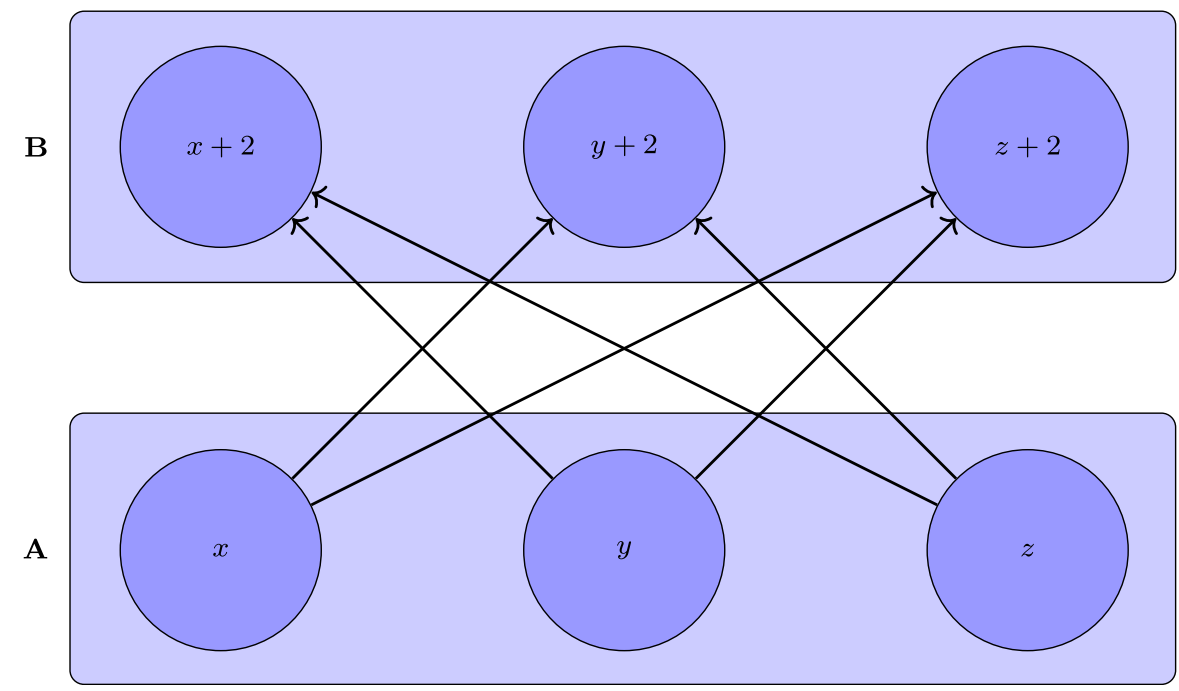

Fig. 3 Representation of a preordered space, defined in Proposition 8, where injective monotones exist and countable multi-utilities do not. In particular, we show $A:=[0,1], B:=[2,3]$ and how $x, y, z \in A$, $x<y<z$, are related to $x+2, y+2, z+2 \in B$. Notice an arrow from an element $w$ to an element $t$ represents $w \prec t$ 
necessary conditions for the existence of monotones, which could collectively be described by the term order separability. Many important results from mathematical economics fall into this category, such as the Debreu Open Gap Lemma (Debreu, 1964), the Nachbin Separation Theorem (Nachbin, 1965), Szpilrajn's theorem (Szpilrajn, 1930), and Fishburn's theorem (Fishburn, 1970, Theorem 3.1). We discuss the role of injective monotones relative to order separability in the following section.

\section{Relating monotones to order separability}

A subset $Z \subseteq X$, such that $x \prec y$ implies that there exists $z \in Z$ with $x \prec z \prec y$ is called order dense (Bridges \& Mehta, 2013; Ok, 2002), and $Z$ is called order dense in the sense of Debreu (or Debreu dense for short) if $x \preceq z \preceq y$. Accordingly, we say that $(X, \preceq)$ is order separable if there exists a countable order dense set (Mehta, 1986a), and Debreu separable if there exists a countable Debreu dense set in $(X, \preceq)$. Notice, our definition of order separability is also known as weak separability (Ok, 2002).

It is well known that a total preorder $\preceq$ has a utility function if and only if it is Debreu separable (e.g., Bridges \& Mehta, 2013, Theorem 1.4.8). Moreover, if $\preceq$ is non-total, then Debreu separability still implies the existence of strict monotones (Bridges \& Mehta, 2013; Debreu, 1954; Herden \& Levin, 2012). The converse, however, is not true, i.e., there are preordered spaces with strict monotones that are not Debreu separable. For example, any Debreu dense subset of $\left(\mathbb{P}_{\Omega}, \preceq_{U}\right)$ is uncountable (if $|\Omega|>2$ )—see Appendix A.1 for a proof. While Debreu separability is concerned with elements satisfying $x \prec y$, an analogous condition that is sufficient for the existence of injective monotones must also consider incomparable elements.

We call a subset $Z \subseteq X$ upper dense if $x \bowtie y$ implies that there exists a $z \in Z$ such that $x \bowtie z \prec y$, and it is called upper dense in the sense of Debreu (or Debreu upper dense for short) if $x \bowtie z \preceq y$. Accordingly, $(X, \preceq)$ is called upper separable if there exists a countable subset of $X$ which is both order dense and upper dense (Ok, 2002), and $(X, \preceq)$ is called Debreu upper separable if there exists a countable subset which is both Debreu dense and Debreu upper dense. We list all mentioned order denseness and separability properties in Table 1.

Proposition 9 If $(X, \preceq)$ is a Debreu upper separable preordered space, then there exists a countable multi-utility; in particular, there exists an injective monotone.

Proof Consider a countable set $D$ given by Debreu upper separability. We will show

\footnotetext{
${ }^{4}$ Notice that, for a fixed pair $x, y \in X$ where $x \bowtie y$ holds, if $Z \subseteq X$ is upper dense, there exist $z_{1}, z_{2} \in Z$ such that $x \bowtie z_{1} \prec y$ and $y \bowtie z_{2} \prec x$. The same applies to upper density in the sense of Debreu, substituting $\prec$ by $\preceq$.
} 
Table 1 Separability properties of preordered spaces $(X, \preceq)$

\begin{tabular}{lll}
\hline Name & Object & Definition \\
\hline Order dense & $Z \subseteq X$ & $\forall x, y \in X x \prec y \Rightarrow \exists z \in Z: x \prec z \prec y$ \\
Debreu dense & $Z \subseteq X$ & $\forall x, y \in X x \prec y \Rightarrow \exists z \in Z: x \preceq z \preceq y$ \\
Upper dense & $Z \subseteq X$ & $\forall x, y \in X x \bowtie y \Rightarrow \exists z \in Z: x \bowtie z \prec y$ \\
Debreu upper dense & $Z \subseteq X$ & $\forall x, y \in X x \bowtie y \Rightarrow \exists z \in Z: x \bowtie z \preceq y$ \\
Order separable & $X$ & $\exists Z \subseteq X$ countable: $Z$ is order dense \\
Debreu separable & $X$ & $\exists Z \subseteq X$ countable: $Z$ is Debreu dense \\
Upper separable & $X$ & $\exists Z \subseteq X$ countable: $Z$ is order dense and upper dense \\
Debreu upper separable & $X$ & $\exists Z \subseteq X$ countable: $Z$ is Debreu dense and Debreu upper dense \\
\hline
\end{tabular}

$$
x \preceq y \Longleftrightarrow\left\{\begin{array}{l}
\chi_{i(d)}(x) \leq \chi_{i(d)}(y) \\
\chi_{r(d)}(x) \leq \chi_{r(d)}(y)
\end{array} \forall d \in D,\right.
$$

where $i(d):=\{y \in X \mid d \preceq y\}$ and $r(d):=\{y \in X \mid d \prec y\}$. By transitivity $x \preceq y$ implies $\chi_{i(d)}(x) \leq \chi_{i(d)}(y)$ and $\chi_{r(d)}(x) \leq \chi_{r(d)}(y) \forall d \in D$. If $\neg(x \preceq y)$ then either $y \prec x$ or $y \bowtie x$. If $y \prec x$, then there exists some $d \in D$ such that either $\chi_{i(d)}(x)>\chi_{i(d)}(y)$ or $\chi_{r(d)}(x)>\chi_{r(d)}(y)$. If $y \bowtie x$ then there exists some $d \in D$ such that $y \bowtie d \preceq x$ which means $\chi_{i(d)}(x)>\chi_{i(d)}(y)$. Since there exists a countable multi-utility, as we just showed, there is an injective monotone by Proposition 5.

Since Debreu upper separability still requires a countable Debreu dense set, the converse of Proposition 9 is again false due to the uncertainty preorder not being Debreu separable (Appendix A.1). However, as can be seen from the proof, if we remove Debreu denseness as a requirement, i.e., if we only require $D$ to be Debreu upper dense, then the only part of the proof that does not work is to follow from $y \prec x$ that there exists an element $v$ of the multi-utility with $v(x)>v(y)$. Since a strict monotone has exactly this property, we obtain the following proposition.

Proposition 10 Consider $(X, \preceq)$ a preordered space. If there exists a countable Debreu upper dense set, then the following are equivalent:

(i) There exists a strict monotone.

(ii) There exists an injective monotone.

(iii) There exists a countable multi-utility.

Proof Assume there exists a countable Debreu upper dense set $D \subseteq X$. It is enough to show that $(i)$ implies (iii), which follows along the same lines as the proof of Proposition 9, but with the multi-utility consisting of $\{u\} \cup\left\{\chi_{i(d)}\right\}_{d \in D}$, where $u$ is a strict monotone. 


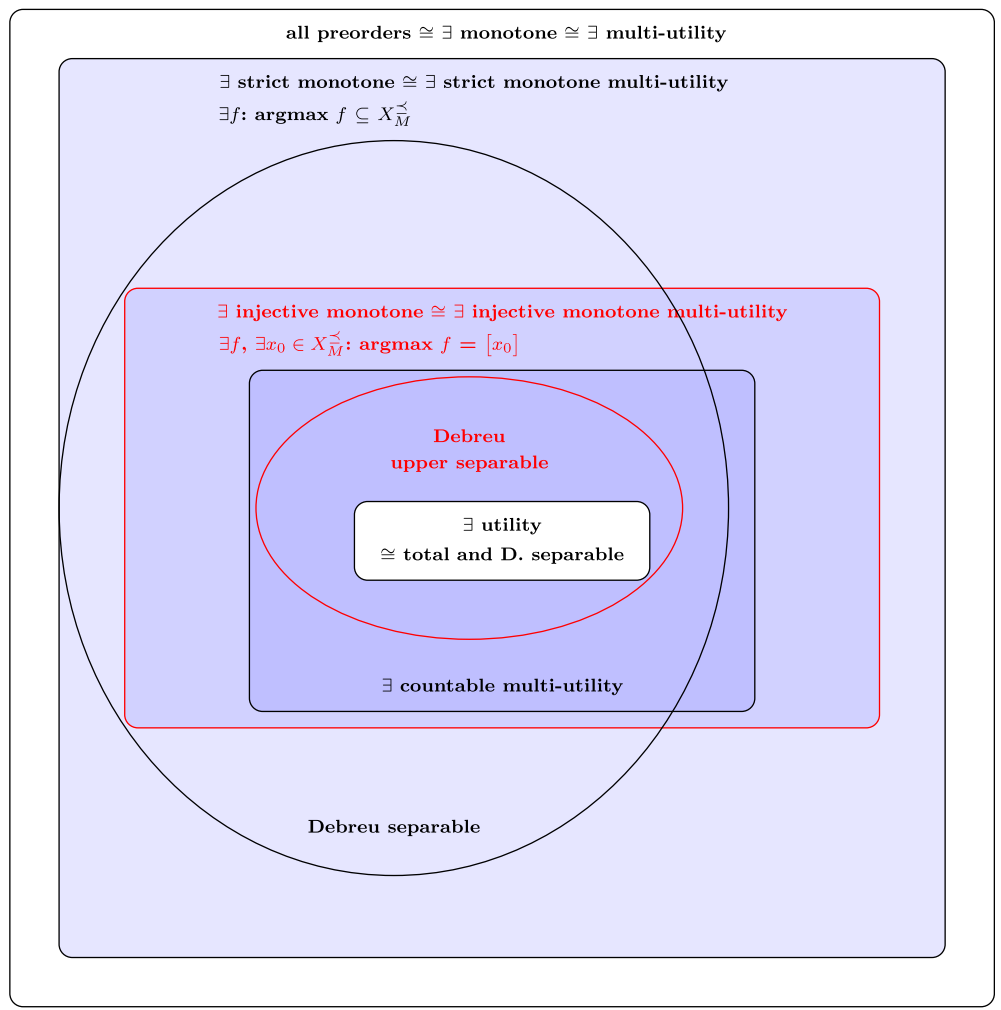

Fig. 4 Classification of preordered spaces $(X, \preceq)$ in terms of representations by real-valued functions (boxes) and order properties (ellipses). We include known relations in black and our contributions in red. Notice, by Proposition 10, the blue area is empty whenever there exists a countable Debreu upper dense set in $X$

The situation in Proposition 10 corresponds exactly to the situation of the uncertainty preorder, which has a countable Debreu upper dense set (Appendix A.1) and, e.g., Shannon entropy as a strict monotone.

\section{Discussion}

In this paper, we are mainly concerned with the introduction of injective monotones, their relation to other monotones, optimization, multi-utilities and order separability, and the application to the uncertainty preorder. The key contributions of our work are the following. First, we refine the classification of preordered spaces based on the existence of monotones. In particular, by extending known results for strict monotones to injective monotones, we find conditions for their existence from different perspectives: other classes of monotones, optimization principles, separating families of increasing sets, and (in particular, countable) multi-utilities. An overview of our conditions in relation to previous work can be found in Fig. 4. Second, we introduce the notion of upper Debreu separability, an order separability 
property that allows to extend well-known results about the existence of monotones on Debreu separable spaces to countable multi-utilities and injective monotones. Finally, we apply our general results to the uncertainty preorder, defined on the space of probability distributions over finite sets, in particular, by establishing order separability properties.

Hierarchy of preordered spaces. A number of scientific disciplines rely on preordered spaces and their representation by monotones, as was already pointed out in Campión et al. (2018), Candeal et al. (2001) and Minguzzi (2010). In Fig. 1, we classify the space of preorders in terms of the existence of certain monotones relevant in various disciplines, which leads to a hierarchy of classes of preordered spaces. The conception of injective monotones then allows for a refinement of this hierarchy of preorders.

Historically, much of the early development of real-valued representations has focused on total preordered spaces that allow for the existence of utility functions. In particular, in the field of mathematical economics, utility theory has pioneered the axiomatic study of conditions that ensure the existence of utility functions for a preordered set $(X, \preceq)$, where $X$ is a set of commodities and $\preceq$ is some total preference relation, a total preorder (Debreu, 1954; Rébillé, 2019). Similarly, we can consider statistical estimation, where the aim is to infer the distribution of a random variable $X$ from some of its realizations. Assuming the distribution belongs to a family $\left\{p_{\theta}\right\}_{\theta \in \mathbb{R}^{N}}$ for some $N>0$, a loss function $\ell: \mathbb{R}^{N} \rightarrow \mathbb{R}$ allows rating distributions according to how well they fit with the observed data: $p_{\theta} \preceq_{\ell} p_{\theta^{\prime}}$ if and only if $-\ell(\theta) \leq-\ell\left(\theta^{\prime}\right)$ where $\theta, \theta^{\prime} \in \mathbb{R}^{N}$ (Hennig \& Kutlukaya, 2007). Choosing a loss function $\ell$ corresponds, thus, to defining a total preorder with a utility representation $\preceq_{\ell}$ on $\left\{p_{\theta}\right\}_{\theta \in \mathbb{R}^{N}}$.

Another example of a preorder with a utility function is equilibrium thermodynamics. Given a thermodynamic system, we consider $\left(X, \preceq_{A}\right)$ where $X$ is the set of all equilibrium states for the system and $x \preceq_{A} y$ if and only if $y$ is adiabatically accessible from $x \forall x, y \in X$ (Lieb \& Yngvason, 1999), that is, one can turn $x$ into $y$ using a device and a weight, with the device returning to its initial configuration at the end and the weight being allowed to change position in some gravitational field. The main concern in the area is the so-called entropy representation problem (Candeal et al., 2001), that is, the existence of a utility function, called entropy function, for $\left(X, \preceq_{A}\right)$ (Lieb \& Yngvason, 1999).

Assuming a total preorder as in the previous examples is necessary for the existence of a utility function, but renders injective monotones uninteresting, as they become equivalent to strict monotones. When the totality assumption is dropped, the classes of preorders with these monotones can be distinguished. A well-known instance of non-total preorders with injective monotones is our running example, the uncertainty preorder. One of its relevant applications lies in the study of quantum entanglement, as it characterizes the possible transformations using local operations and classical communications (Nielsen, 1999, Theorem 1). In physics, the uncertainty preorder given by majorization has recently also been extended. Given $\ell_{1}^{1}\left(\mathbb{R}^{+}\right):=\left\{\left(p_{i}\right)_{i \in \mathbb{N}} \mid 0 \leq p_{i} \leq 1, \sum_{i=1}^{\infty} p_{i}=1\right\}$, we define infinite majorization $\preceq_{I M}$ (Li \& Busch, 2013) for any $p, q \in \ell_{1}^{1}\left(\mathbb{R}^{+}\right)$like 


$$
p \preceq_{I M} q: \Leftrightarrow \sum_{i=1}^{k} p_{i}^{\downarrow} \leq \sum_{i=1}^{k} q_{i}^{\downarrow} \forall k \in \mathbb{N},
$$

where $p \downarrow$ represents $p$ ordered in a decreasing way. Since $\preceq_{I M}$ is defined through a countable multi-utility, there exist injective monotones by Proposition 5. Finally, the uncertainty preorder is also an instance of multicriteria optimization (Ehrgott, 2005), also known as vector optimization (Jahn, 2009), since it is concerned with the simultaneous optimization of a finite number of objective functions (1). Notice strict and injective monotones belong to the scalarization techniques (Bevilacqua et al., 2018b; Ehrgott, 2005; Jahn, 2009) in vector optimization and always exist, again by Proposition 5 .

Preordered spaces from the next general class, the ones with strict monotones, include general relativity. Spacetime can be studied as a pair $\left(M, \preceq_{C}\right)$ where $M$ is a set of events and $\preceq_{C}$ is a causal relation, a partial order specifying which events can influence others, which lie to the future of others (Bombelli et al., 1987). A usual question is to establish sufficient conditions on $\left(M, \preceq_{C}\right)$ for the existence of strict monotones, which are referred to as time functions (Minguzzi, 2010) and are usually required to be continuous according to some topology. The study of physically plausible conditions from which countable multi-utilities or injective monotones can be constructed has, to our knowledge, not been addressed yet in the field. Notice, spacetime was originally approached through a differentiable structure $(M, g)$, where $M$ is a manifold and $g$ a metric, and was only later studied as a partial order (Bombelli et al., 1987).

Another case of preorders with strict monotones is trumping. Consider $\left(\mathbb{P}_{\Omega}, \preceq_{T}\right)$ the space of probability distributions over some finite set $\Omega, \mathbb{P}_{\Omega}$, with the trumping preorder

$$
p \preceq_{T} q \Longleftrightarrow \exists r \in \mathbb{P}_{\Omega^{\prime}}\left|\Omega^{\prime}\right|<\infty \text { s.t. } p \otimes r \preceq_{M} q \otimes r,
$$

where $p \otimes r:=\left(p_{1} r_{1}, . ., p_{1} r_{\Omega^{\prime}}, . ., p_{\Omega} r_{1}, . ., p_{\Omega} r_{\Omega^{\prime}}\right) \quad \forall p \in \mathbb{P}_{\Omega}, r \in \mathbb{P}_{\Omega^{\prime}}$ and $\left|\Omega^{\prime}\right|<\infty$ (Müller \& Pastena, 2016). Trumping extends majorization taking into account transformations using a third state, a catalyst. As an example, consider $p:=(0.4,0.4,0.1,0.1), q:=(0.5,0.25,0.25,0)$ and $r:=(0.6,0.4)$. Notice $\neg\left(p \preceq_{M}\right.$ $q$ ) but $p \otimes r \preceq_{M} q \otimes r$, implying $p \preceq_{T} q$. Questions regarding physically meaningful strict monotones and multi-utilities for trumping are relevant (Turgut, 2007). As no countable multi-utility has been found, it remains a question whether injective monotones do exist.

A final example from the most general class of preorders, the one where only monotones exist, are social welfare relations (SWR) in economics. A SWR is a partial order $\preceq_{S}$ defined on the countably infinite product of the unit interval $X:=\prod_{n \in \mathbb{N}}[0,1]$. A SWR is said to be ethical if (1) given $x, y \in X$ with some $i, j \in \mathbb{N}$ such that $x_{i}=y_{j}, y_{i}=x_{j}$ and $x_{k}=y_{k} \forall k \notin\{i, j\}$ we have $x \sim \sim_{s} y$ and (2) given $x, y \in X$ where $x_{i} \leq y_{i} \forall i \in \mathbb{N}$ and $x_{j}<y_{j}$ for some $j \in \mathbb{N}$ then $x \prec_{S} y$. Any ethical SWR is an example of a preordered space without strict monotones (Banerjee \& Dubey, 2010, Proposition 1) and, thus, without both injective monotones and countable multi-utilities. 
Monotones and topology. While we have focused on preordered spaces and left some brief comments regarding topology for Appendix A.3, in the past they have been often studied together. The original interest in functions representing order structures was concerned with (continuous) utility representations of total topological preordered spaces (Debreu, 1954, 1964; Eilenberg, 1941). Of particular importance were results concerning the existence of a continuous utility function for both connected and separable total topological preordered spaces (Eilenberg, 1941) and for second countable total topological preordered spaces (Debreu, 1954). Among the classical results we also find the existence of an order isomorphism between a subset of the real numbers and any total order with countably many jumps whose order topology is second countable (Fleischer, 1961). Based on the work of Nachbin (1965) relating topology and order theory, in particular a generalization of Urysohn's separation theorem, the classical results where reproved and sometimes generalized for example in Bosi et al. (2020b), Herden (1989) and Mehta (1977, 1986a, b, 1988).

Multi-utility representations. The study of non-total order structures was introduced in Aumann (1962). Representation of non-total preorders by multiutilities came later and was remarkably developed in Evren and Ok (2011). Although strict monotones can be traced back to Peleg (1970) and Richter (1966), there continue to be advances in the field (Bosi et al., 2020a; Herden \& Levin, 2012; Rébillé 2019). In fact, it was only recently in Minguzzi (2013) where strict monotone multi-utilities were introduced and later in Alcantud et al. (2013, 2016) where they were further studied. The relation of these ideas with optimization and the existence of maximal elements is also present in the literature (Bevilacqua et al., 2018a, b; Bosi \& Zuanon, 2017; Bosi et al., 2018; White, 1980). Countable multiutilities where studied particularly in Alcantud et al. (2016), Bevilacqua et al. (2018c), while finite multi-utility representations were notably advanced in Kaminski (2007) and Ok (2002) and, in vector optimization, in Jahn (2009).

Open questions. While we have shown the existence of injective monotones for the widely studied class of preorders with countable multi-utilities, our construction is impractical since it relies on an infinite sum. For specific applications, injective monotones with a simpler representation are of interest. In general, any of the disciplines where these ideas are applied would benefit from a better understanding of the classification of preordered spaces in terms of real-valued monotones. For example, regarding the maximum entropy principle, the classification could be useful to reconsider the reasoning behind the choice of Shannon entropy. Even though there have been many principled approaches to "derive" Shannon entropy as a measure of uncertainty in the past, such as Aczél et al. (1974) and Shore and Johnson (1980), and for many practical purposes its appealing properties overweigh the bias in choosing this particular strict monotone, the question remains whether one should maximize entropy or maximize uncertainty. Quantum physics could also benefit as, for instance, the preorder underlying entanglement catalysis, trumping, is not well understood (Müller \& Pastena, 2016). Many relevant open questions related to our work can also be found in Bosi et al. (2020a), for example, while we have focused mostly on preordered spaces and made some remarks on semicontinuity, it would be important to study continuous injective monotones in terms of 
topological properties of the underlying spaces, as in the classical works on utility functions.

\section{Appendix}

\section{Entropy and the uncertainty preorder}

In the following, we provide proofs for statements regarding the uncertainty preorder $\preceq_{U}$ and entropy $H$ that appear throughout the main part of this article, in particular, all results are stated with respect to the preordered space $\left(\mathbb{P}_{\Omega}, \preceq_{U}\right)$, for a finite set $\Omega$.

\section{Lemma 4 (Basic facts)}

(i) Shannon entropy is a strict monotone. If $|\Omega| \geq 3$ then it is not an injective monotone.

(ii) If $|\Omega| \geq 3$ then for all $c \in(0, \log |\Omega|)$, there is an uncountable set $S_{c}$ such that $H(s)=c \forall s \in S_{c}$. In particular, there are $p, q \in \mathbb{P}_{\Omega}$ with $c=H(p)=H(q)$ but $p \bowtie q$ for all $c \in(0, \log |\Omega|)$.

\section{Proof}

(i) Strict monotonicity of $H$ comes from the fact $H(p)=\sum_{i=1}^{\mid \Omega} f\left(p_{i}\right)$ where $f(x)=-x \log (x)$ is a strictly convex function. Given any other strictly convex $f$, strict monotonicity will still hold. One can find the details in (Marshall et al., 1979, C.1.a). $H$ is not an injective monotone for $|\Omega| \geq 3$ by (ii).

(ii) Given $p, q \in \mathbb{P}_{\Omega}$, we denote by $\overline{p q}$ the segment with endpoints $p$, $q$. Consider $u \in \mathbb{P}_{\Omega}$ the uniform distribution, $e_{i}, e_{j} \in \mathbb{P}_{\Omega}$ Dirac distributions for two different elements $i, j \in \Omega$ and some $c \in(0, \log |\Omega|)$. Consider some $c^{\prime}$ s.t. $0<c^{\prime}<\min \{c, H(m)\}$ where $m$ is the middle point of $\overline{e_{i} e_{j}}$. By the intermediate value theorem, there exists some $r \in \overline{e_{i} m}$ such that $H(r)=c^{\prime}$. Consider now a parametrization of $\overline{e_{i} r}:\left\{r_{t}\right\}_{t \in[0,1]}$ and define $\ell_{t}:=\overline{r_{t} u}$ for each $t \in[0,1]$. Again by the intermediate value theorem, since $H\left(r_{t}\right)<c \forall t \in[0,1]$, there exists some $p_{t} \in \ell_{t}$ such that $H\left(p_{t}\right)=c \forall t \in[0,1]$. By construction, given $t, t^{\prime} \in[0,1] t \neq t^{\prime}$ we have $p_{t} \neq p_{t^{\prime}}$ since $\ell_{t} \cap \ell_{t^{\prime}}=\{u\}$ whenever $t \neq t^{\prime}$ which means $\left\{p_{t}\right\}_{t \in[0,1]}$ is uncountable. In particular, there are $t_{c}, t_{c}^{\prime} \in[0,1] t_{c} \neq t_{c}^{\prime}$ such that $p_{t_{c}} \bowtie p_{t_{c}^{\prime}}$ and $H\left(p_{t_{c}}\right)=H\left(p_{t_{c}^{\prime}}\right)=c$ for for every $c \in(0, \log |\Omega|)$. 


\section{Lemma 5 (Debreu separability)}

(i) If $|\Omega|=2$ then $\left(\mathbb{P}_{\Omega}, \preceq_{U}\right)$ is order separable. In particular, $\left(\mathbb{P}_{\Omega}, \preceq_{U}\right)$ is Debreu separable for $|\Omega|=2$.

(ii) If $|\Omega| \geq 3$ then any subset $Z \subseteq \mathbb{P}_{\Omega}$ which is Debreu dense in $\left(\mathbb{P}_{\Omega}, \preceq_{U}\right)$ has the cardinality of the continuum $|Z|=\mathfrak{c}$.

(iii) For any $|\Omega|<\infty$, there exists a countable upper dense set $Z \subseteq \mathbb{P}_{\Omega}$.

Proof For simplicity of notation, in the following, we omit the subscript $U$ and thus write $\preceq$ for $\preceq_{U}$ (analogously for $\bowtie$ and $\prec$ ).

(i) Consider $p, q \in \mathbb{P}$ such that $p \prec q$. By definition, we have $q_{1}^{\downarrow}<p_{1}^{\downarrow}$. Consider some $s \in \mathbb{Q}$ such that $q_{1}^{\downarrow}<s<p_{1}^{\downarrow}$. Notice by normalization $\frac{1}{2} \leq q_{1}^{\downarrow}<s$ and by normalization again $1-s<s$. Thus, $p \prec r \prec q$ where $r^{\downarrow}:=(s, 1-s)$ and $\mathbb{Q}^{2} \cap \mathbb{P}_{\Omega}$ is countable and order dense in $\left(\mathbb{P}_{\Omega}, \preceq_{U}\right)$ for $|\Omega|=2$. In particular, $\left(\mathbb{P}_{\Omega}, \preceq\right)$ is Debreu separable for $|\Omega|=2$ which we could have known applying Theorem 1.4.8 in Bridges and Mehta (2013) since for $|\Omega|=2$ there is a utility function, $u_{1}$.

(ii) Fix $|\Omega|=3$. Consider for some $x \in\left(\frac{1}{2}, 1\right)$ some $p \in \mathbb{P}_{\Omega}$ such that $p_{1}^{\downarrow}=x$. Notice $p_{2}^{\downarrow}+p_{3}^{\downarrow}<x$ by normalization. Take some $\epsilon>0$ such that $p_{2}^{\downarrow}+\epsilon<x$ and $\epsilon \leq p_{3}^{\downarrow}$ to define $q \in \mathbb{P}_{\Omega}$ where $q^{\downarrow}:=\left(x, p_{2}^{\downarrow}+\epsilon, p_{3}^{\downarrow}-\epsilon\right)$. Notice $q \prec p$. Notice for any $x \in\left(\frac{1}{2}, 1\right)$ we can define a pair $q_{x}, p_{x} \in \mathbb{P}_{\Omega}$ such that $q_{x} \prec p_{x}$ as we did before where for any $t \in \mathbb{P}_{\Omega}$ such that $q_{x} \preceq t \preceq p_{x}$ we have $t_{1}^{\downarrow}=x$. Given $Z \subseteq \mathbb{P}_{\Omega}$ a subset which is Debreu dense in $\left(\mathbb{P}_{\Omega}, \preceq\right)$ there exists for any $x \in\left(\frac{1}{2}, 1\right)$ some $z_{x} \in Z$ such that $q_{x} \preceq z_{x} \preceq p_{x}$. Fix for every $x \in\left(\frac{1}{2}, 1\right)$ some $z_{x}$. Notice, given $x, y \in\left(\frac{1}{2}, 1\right)$, then $z_{x}=z_{y}$ implies $x=\left(z_{x}\right)_{1}^{\downarrow}=\left(z_{y}\right)_{1}^{\downarrow}=y$ which means that $\varphi:\left(\frac{1}{2}, 1\right) \rightarrow Z, x \mapsto z_{x}$ is injective, implying $\mathfrak{c} \leq|Z|$. Since $Z \subseteq \mathbb{P}_{\Omega}$ and $\left|\mathbb{P}_{\Omega}\right|=\mathfrak{c}$ we have $|Z|=\mathfrak{c}$. In case $|\Omega|>3$, any Debreu dense subset would also be Debreu dense in the subset with $|\Omega|=3$. We can thus follow the above lines and get the same conclusion for any $|\Omega| \geq 3$. 
(iii) Consider $x, y \in \mathbb{P}_{\Omega}$ such that $x \bowtie y$. Since $x \bowtie y$, there exist $n, m \leq|\Omega|-1$ such that $\sum_{i=1}^{n} x_{i}^{\downarrow}<\sum_{i=1}^{n} y_{i}^{\downarrow}$ and $\sum_{i=1}^{m} x_{i}^{\downarrow}>\sum_{i=1}^{m} y_{i}^{\downarrow}$. Notice $y_{i}^{\downarrow}<1 \quad \forall i \leq|\Omega|$ since in the opposite case $y \preceq x \forall x \in \mathbb{P}_{\Omega}$. Consider $1<k \leq|\Omega|$ the largest integer such that $y_{k}^{\downarrow}>0$ and define $\left\{\epsilon_{i}\right\}_{i=1}^{k-1}$ where

$$
\left\{\begin{array}{cc}
0<\epsilon_{i}<\min \left\{y_{k}^{\downarrow}, \quad \sum_{j=1}^{m} x_{j}^{\downarrow}-\sum_{j=1}^{m} y_{j}^{\downarrow}\right\} & \text { if } i=1, \\
0<\epsilon_{i}<\min \left\{y_{k}^{\downarrow}-\sum_{j=1}^{i-1} \epsilon_{j}, \quad \sum_{j=1}^{m} x_{j}^{\downarrow}-\sum_{j=1}^{m} y_{j}^{\downarrow}-\sum_{j=1}^{i-1} \epsilon_{j}\right\} & \text { if } 1<i \leq m, \\
0<\epsilon_{i}<y_{k}^{\downarrow}-\sum_{j=1}^{i-1} \epsilon_{j} & \text { if } m<i<k .
\end{array}\right.
$$

Notice $m<k$ since the opposite case leads to $\sum_{i=1}^{m} x_{i}^{\downarrow}>\sum_{i=1}^{m} y_{i}^{\downarrow}=$ $\sum_{i=1}^{k} y_{i}^{\downarrow}=1$ contradicting normalization. For all $i<k$ choose $q_{i} \in\left(y_{i}^{\downarrow}, y_{i}^{\downarrow}+\right.$ $\left.\epsilon_{i}\right) \cap \mathbb{Q}$ such that $q_{i} \geq q_{i+1} \quad$ and $q_{k}=1-\sum_{i=1}^{k-1} q_{i}$. Then $z:=$ $\left(q_{1}, q_{2}, . ., q_{k}, 0, . ., 0\right)$ has $|\Omega|-k$ zeros, the same number of zeros as $y$, and $z=z^{\downarrow}$, since $q_{k}<1-\sum_{i=1}^{k-1} y_{i}^{\downarrow}=y_{k} \leq y_{k-1}<q_{k-1}$. By construction, we have $\sum_{j=1}^{i} z_{j}^{\downarrow}>\sum_{j=1}^{i} y_{j}^{\downarrow} \forall i<k$ implying $\sum_{j=1}^{n} x_{j}^{\downarrow}<\sum_{j=1}^{n} z_{j}^{\downarrow}, \sum_{j=1}^{|\Omega|} z_{j}^{\downarrow}=\sum_{j=1}^{k} z_{j}^{\downarrow}=$ 1 and $\sum_{j=1}^{m} z_{j}^{\downarrow}<\sum_{j=1}^{m} x_{j}^{\downarrow}$ since

$$
\sum_{j=1}^{m} z_{j}^{\downarrow}<\sum_{j=1}^{m} y_{j}^{\downarrow}+\epsilon_{j}<\sum_{j=1}^{m} y_{j}^{\downarrow}+\sum_{j=1}^{m} x_{j}^{\downarrow}-\sum_{j=1}^{m} y_{j}^{\downarrow}=\sum_{j=1}^{m} x_{j}^{\downarrow}
$$

where in the first inequality, we applied $z_{j}^{\downarrow}=q_{j}<y_{j}^{\downarrow}+\epsilon_{j} \forall j \leq m$ and in the second, we applied the definition of $\epsilon_{m}$ by which $\sum_{j=1}^{m} \epsilon_{j}<\sum_{j=1}^{m} x_{j}^{\downarrow}-\sum_{j=1}^{m} y_{j}^{\downarrow}$. Thus, $x \bowtie z \prec y$. We have shown $\mathbb{Q}^{|\Omega|} \cap \mathbb{P}_{\Omega}$ is a countable upper dense set in $\left(\mathbb{P}_{\Omega}, \preceq_{U}\right)$ for any $|\Omega|<\infty$.

\section{Proofs}

\section{Preorders without strict monotones (Alcantud et al., 2016, Corollary 2.2)}

For example, consider the power set of the reals equipped with set inclusion, $(\mathcal{P}(\mathbb{R}), \subseteq)$. Since $\subseteq$ is reflexive, transitive, and antisymmetric (i.e., a partial order), by Szpilrajn extension theorem, there exists a totally ordered space $(\overline{\mathcal{P}(\mathbb{R})}, \preceq)$ extending $(\mathcal{P}(\mathbb{R}), \subseteq)$, respecting the relations that already exist and relating the incomparable elements (e.g., overlapping intervals). Hence, if there was a strict monotone $v: \overline{\mathcal{P}(\mathbb{R})} \rightarrow \mathbb{R}$, then $v(U)=v(V)$ for some $U, V \subseteq \mathbb{R}$ would imply that $U=V$, because w.l.o.g. $U \preceq V$, and $U \prec V$ cannot hold since $v$ is a strict monotone. This contradicts Cantor's theorem by which the cardinality of the power set $\mathcal{P}(\mathbb{R})$ is strictly greater than that of $\mathbb{R}$. 


\section{Proof of Lemma 1}

First, note that for $r \in(0,1)$, we have

$$
r \in\left(0, \frac{1}{2}\right) \Longleftrightarrow r^{m}>\sum_{n=m+1}^{\infty} r^{n} \forall m \geq 0 .
$$

This is a direct consequence of the closed-form formula of the geometric series and its partial sums, by which we have for any $r \in(0,1)$ and $m \geq 0$,

$$
\sum_{n=m+1}^{\infty} r^{n}=\frac{1}{1-r}-\sum_{n=0}^{m} r^{n}=\frac{r}{1-r} r^{m},
$$

so that $r^{m}>\sum_{n=m+1}^{\infty} r^{n}$ if and only if $r<1-r$, i.e., $r \in\left(0, \frac{1}{2}\right)$.

Consider $x, y \in X$ and $m \in \mathbb{N}$ be the smallest index such that $\chi_{A_{m}}(x) \neq \chi_{A_{m}}(y)$. Assume $\chi_{A_{m}}(x)<\chi_{A_{m}}(y)$, in particular $\chi_{A_{m}}(x)=0$ and $\chi_{A_{m}}(y)=1$. Then,

$$
\begin{aligned}
c(x) & \leq \sum_{n=0}^{m} r^{n} \chi_{A_{n}}(x)+\sum_{n=m+1}^{\infty} r^{n} \\
& \stackrel{(a)}{<} \sum_{n=0}^{m} r^{n} \chi_{A_{n}}(x)+r^{m} \stackrel{(b)}{=} \sum_{n=0}^{m-1} r^{n} \chi_{A_{n}}(y)+r^{m} \chi_{A_{m}}(y) \leq c(y)
\end{aligned}
$$

where $(a)$ is due to (7) and $(b)$ follows from the choice of $m$. Conversely, if $c(x)<c(y)$, then let $m$ be the first index where $\chi_{A_{m}}(x) \neq \chi_{A_{m}}(y)$. Clearly, if $\chi_{A_{m}}(y)<\chi_{A_{m}}(x)$, then by the same argument as above, $c(y)<c(x)$, contradicting the hypothesis. Hence, $\chi_{A_{m}}(x)<\chi_{A_{m}}(y)$.

\section{Proof of Lemma 3}

Given a family of increasing sets $\left(A_{n}\right)_{n \in \mathbb{N}}, c$ in (3) is a monotone. If $x \prec y$, then by assumption, there exists $n \in \mathbb{N}$ such that $x \notin A_{n}$ and $y \in A_{n}$, i.e., $\chi_{A_{n}}(x)<\chi_{A_{n}}(y)$, implying that $c$ is a strict monotone by Lemma 1. Furthermore, if $x \bowtie y$, there exists $n \in \mathbb{N}$ such that $x \notin A_{n}$ and $y \in A_{n}$ or $y \notin A_{n}$ and $x \in A_{n}$. There exists thus some $m \in \mathbb{N}$ such that $\chi_{A_{m}}(x) \neq \chi_{A_{m}}(y)$ implying $c(x) \neq c(y)$ by Lemma 1. By Lemma 2, we have $c$ is an injective monotone.

\section{Proof of Proposition 7}

(i) We only need to show given a strict monotone $v$ there exists a countable set $\left(A_{n}\right)_{n \in \mathbb{N}}$ that separates any pair $x, y \in X$ such that $x \prec y$, the converse is true by Lemma 3 Consider $A_{n}:=v^{-1}\left(\left[q_{n}, \infty\right)\right)$ where $\left(q_{n}\right)_{n \in \mathbb{N}}$ is a numeration of the rational numbers. If $x \prec y$, there exists $q_{n} \in \mathbb{Q}$ such that $v(x)<q_{n}<v(y)$ which means $y \in A_{n}$ and $x \notin A_{n}$. 
(ii) Relying on Lemma 3 and (i), we can take $\left(A_{n}\right)_{n \in \mathbb{N}}$ defined as in (i) assuming $v$ is an injective monotone. Given $x, y \in X$ such that $x \bowtie y$, we have $v(x) \neq$ $v(y)$ which implies there exists some $q_{n} \in \mathbb{Q}$ between $v(x)$ and $v(y)$, i.e., either $x \in A_{n}$ and $y \notin A_{n}$ or $y \in A_{n}$ and $x \notin A_{n}$.

\section{Semicontinuity}

Much of the economic literature on utility representations in preordered spaces is concerned with topological questions, in particular, under which conditions on the preordered space one can expect that monotones and utilities satisfy certain continuity properties (e.g., Alcantud et al., 2016; Debreu, 1964; Mehta, 1986a). This is particularly important for optimization, since continuous functions attain their maximal elements on compact sets. Therefore, in this section, we collect the continuity properties of the injective monotones that appear in the main part of this article.

Given a topology $\tau$, a triple $(X, \preceq, \tau)$ is called a preordered topological space. A function $f:(X, \tau) \rightarrow\left(\mathbb{R}, \tau_{\text {nat }}\right)$, where $\tau_{\text {nat }}$ is the topology given by the Euclidean metric, is said to be upper semicontinuous if $f^{-1}((-\infty, r)) \in \tau \forall r \in \mathbb{R}$.

Upper semicontinuous functions retain the property of continuous functions that they assume their maxima on compact sets, that is, they are effective on any compact set $B \subseteq X$.

Similarly, we say $(X, \preceq, \tau)$ is upper semicontinuous if $i(x)=\{z \in X \mid x \preceq z\}$ is closed $\forall x \in X$. We may abuse notation and say that $\preceq$ is upper semicontinuous whenever $X$ and $\tau$ are clear.

Proposition 11 Let $(X, \preceq, \tau)$ be a preordered topological space.

(i) In Proposition 4, we can choose an upper semicontinuous multi-utility if the monotone is upper semicontinuous.

(ii) In Proposition 5, we can add upper semicontinuous to both the hypothesis and the thesis.

(iii) In Proposition 6, if the preorder is upper semicontinuous then the equivalence remains true if upper semicontinuous is added to all clauses.

(iv) In Proposition 7, the monotones can be chosen to be upper semicontinuous if and only if the separating families consist of closed increasing sets.

(v) In Proposition 10, if the preorder is upper semicontinuous then the equivalence remains true if upper semicontinuous is added to all clauses.

Notice, the uncertainty preorder $\preceq_{U}$ is upper semicontinuous with respect to the Euclidean topology, since 


$$
i(p)=\left\{q \in \mathbb{P}_{\Omega} \mid p \preceq_{u} q\right\}=\bigcap_{i=1}^{|\Omega|-1}\left\{q \in \mathbb{P}_{\Omega} \mid u_{i}(p) \leq u_{i}(q)\right\}=\bigcap_{i=1}^{|\Omega|-1} u_{i}^{-1}\left(\left[u_{i}(p), \infty\right)\right)
$$

where $u_{i}^{-1}\left(\left[u_{i}(p), \infty\right)\right)$ is closed, because all $u_{i}$ are upper semicontinuous.

\section{Proof}

(i) If there exists an upper semicontinuous injective monotone, then we can construct w.l.o.g. an upper semicontinuous injective monotone $c: X \rightarrow(0,1)$. Since $\preceq$ is upper semicontinuous, we know $\chi_{i(x)}$ is upper semicontinuous, and given the fact the class of upper semicontinuous functions is closed under addition by Proposition 1.5.12 in Pedersen (2012), $c_{x}$ in the proof of Proposition 4 is upper semicontinuous $\forall x \in A_{c}$. Thus, $\{c\} \cup$ $\left\{c_{x}\right\}_{x \in A_{c}}$ is an upper semicontinuous injective monotone multi-utility of $(X, \preceq, \tau)$.

(ii) Take $\left(u_{m}\right)_{m \in M},\left(A_{n}\right)_{n \in \mathbb{N}}$ and $c$ defined as in the proof of Proposition 5. If $\left(u_{m}\right)_{m \in M}$ is upper semicontinuous then $\forall n \in \mathbb{N} A_{n}=u_{m_{n}}^{-1}\left(\left[q_{n}, \infty[) \in \tau^{c}\right.\right.$ and $\chi_{A_{n}}(x)$ is upper semicontinuous $\forall n \in \mathbb{N}$. The class of upper semicontinuous function is closed under addition, product by positive scalars and uniform convergence by Proposition 1.5.12 in Pedersen (2012). By the first two $c_{N}:=\sum_{n=0}^{N} 3^{-n} \chi_{A_{n}}$ is upper semicontinuous $\forall N \in \mathbb{N}$ and by the third $c=$ $\lim _{N \rightarrow \infty} c_{N}$ is upper semicontinuous.

(iii) Following (ii), we get $\{c\} \cup\left\{c_{n_{1}, n_{2}}\right\}_{n_{1}<n_{2}}$ defined as in the proof of Proposition 6 consists of upper semicontinuous injective monotones.

(iv) Notice whenever $\left(A_{n}\right)_{n \in \mathbb{N}}$ in the proof of Proposition 7 is defined through an upper semicontinuous function, either a strict monotone or an injective monotone, then $A_{n}$ is closed $\forall n \in \mathbb{N}$. Conversely, we can follow the proof of (ii) to get upper semicontinuity for both a strict monotone and an injective monotone constructed as in Lemma 3.

(v) We again only show (i) implies (iii) in Proposition 10. If $\preceq$ is upper semicontinuous then $\chi_{i(d)}$ is upper semicontinuous $\forall d \in D$ and since we can choose $u$ to be an upper semicontinuous strict monotone by hypothesis we get $\{u\} \bigcup\left\{\chi_{i(d)}\right\}$ is an upper semicontinuous countable multi-utility.

Funding Open Access funding enabled and organized by Projekt DEAL. This research was funded by the European Research Council, grant number ERC-StG-2015-ERC, Project ID: 678082, "BRISC: Bounded Rationality in Sensorimotor Coordination".

Availability of data and material Not applicable.

Code availability Not applicable. 


\section{Declarations}

Conflict of interest The authors declare that they have no conflict of interest.

Open Access This article is licensed under a Creative Commons Attribution 4.0 International License, which permits use, sharing, adaptation, distribution and reproduction in any medium or format, as long as you give appropriate credit to the original author(s) and the source, provide a link to the Creative Commons licence, and indicate if changes were made. The images or other third party material in this article are included in the article's Creative Commons licence, unless indicated otherwise in a credit line to the material. If material is not included in the article's Creative Commons licence and your intended use is not permitted by statutory regulation or exceeds the permitted use, you will need to obtain permission directly from the copyright holder. To view a copy of this licence, visit http:// creativecommons.org/licenses/by/4.0/.

\section{References}

Aczél, J., Forte, B., \& Ng, C. T. (1974). Why the shannon and hartley entropies are 'natural'. Advances in Applied Probability, 6(1), 131-146. https://doi.org/10.2307/1426210.

Alcantud, J. C. R., Bosi, G., \& Zuanon, M. (2013). Representations of preorders by strong multi-objective functions. Tech. Rep. MPRA Paper 5232, University Library of Munich.

Alcantud, J. C. R., Bosi, G., \& Zuanon, M. (2016). Richter-Peleg multi-utility representations of preorders. Theory and Decision, 80(3), 443-450.

Arnold, B. C. (2018). Majorization and the Lorenz order with applications in applied mathematics and economics. Springer.

Aumann, R. J. (1962). Utility theory without the completeness axiom. Econometrica: Journal of the Econometric Society, 445-462.

Banerjee, K., \& Dubey, R. S. (2010). On multi-utility representation of equitable intergenerational preferences. In Econophysics and Economics of Games, Social Choices and Quantitative Techniques (pp. 175-180). Springer.

Bernoulli, J. (1713). Ars conjectandi. Thurneysen Brothers.

Bevilacqua, P., Bosi, G., \& Zuanon, M. (2018a). Maximal elements of preorders from maximization of transfer upper continuous weak utilities on a compact space. Far East J. Math. Sci, 103, 213-221.

Bevilacqua, P., Bosi, G., \& Zuanon, M. (2018b). Multiobjective optimization, scalarization, and maximal elements of preorders. In Abstract and applied analysis (vol. 2018). Hindawi.

Bevilacqua, P., Bosi, G., \& Zuanon, M. (2018c). Representation of a preorder on a topological space by a countable family of upper semicontinuous order-preserving functions. Advances and Applications in Mathematical Sciences, 17(5), 417-427.

Bombelli, L., Lee, J., Meyer, D., \& Sorkin, R. D. (1987). Space-time as a causal set. Physical Review Letters, 59(5), 521.

Bosi, G., Bevilacqua, P., \& Zuanon, M. (2018). Upper semicontinuous representability of maximal elements for non total preorders on compact spaces. Res J Econ, 3, 2.

Bosi, G., Campión, M. J., Candeal, J. C., \& Indurain, E. (2020). Mathematical topics on representations of ordered structures and utility theory. Springer.

Bosi, G., Estevan, A., \& Raventós-Pujol, A. (2020). Topologies for semicontinuous Richter-Peleg multiutilities. Theory and Decision, 88(3), 457-470.

Bosi, G., \& Zuanon, M. (2013). Existence of maximal elements of semicontinuous preorders. Int J Math Anal, 7, 1005-1010.

Bosi, G., \& Zuanon, M. E. (2017). Maximal elements of quasi upper semicontinuous preorders on compact spaces. Economic Theory Bulletin, 5(1), 109-117.

Brandao, F., Horodecki, M., Ng, N., Oppenheim, J., \& Wehner, S. (2015). The second laws of quantum thermodynamics. Proceedings of the National Academy of Sciences, 112(11), 3275-3279.

Bridges, D. S., \& Mehta, G. B. (2013). Representations of preferences orderings (Vol. 422). Springer Science \& Business Media.

Campión, M. J., Gómez-Polo, C., Induráin, E., \& Raventós-Pujol, A. (2018). A survey on the mathematical foundations of axiomatic entropy: Representability and orderings. Axioms, 7(2), 29. 
Candeal, J. C., De Miguel, J. R., Induráin, E., \& Mehta, G. B. (2001). Utility and entropy. Economic Theory, 17(1), 233-238.

Csiszár, I. (2008). Axiomatic characterizations of information measures. Entropy, 10(3), 261-273.

Dalton, H. (1920). The measurement of the inequality of incomes. The Economic Journal, 30(119), 348-361.

Debreu, G. (1954). Representation of a preference ordering by a numerical function. Decision Processes, 3, 159-165.

Debreu, G. (1964). Continuity properties of paretian utility. International Economic Review, 5(3), 285-293.

Ehrgott, M. (2005). Multicriteria optimization (Vol. 491). Springer Science \& Business Media.

Eilenberg, S. (1941). Ordered topological spaces. American Journal of Mathematics, 63(1), 39-45.

Evren, Ö., \& Ok, E. A. (2011). On the multi-utility representation of preference relations. Journal of Mathematical Economics, 47(4-5), 554-563.

Fishburn, P. C. (1970). Utility theory for decision making. Tech. rep., Research analysis corp McLean VA.

Fleischer, I. (1961). Numerical representation of utility. Journal of the Society for Industrial and Applied Mathematics, 9(1), 48-50.

Fox, R., Pakman, A., \& Tishby, N. (2016). Taming the noise in reinforcement learning via soft updates. In Proceedings of the thirty-second conference on uncertainty in artificial intelligence (pp. 202-211).

Gottwald, S., \& Braun, D. A. (2019). Bounded rational decision-making from elementary computations that reduce uncertainty. Entropy, 21(4), 375.

Gottwald, S., \& Braun, D. A. (2020). The two kinds of free energy and the Bayesian revolution. PLoS Computational Biology, 16(12), e1008420.

Hardy, G. H., Littlewood, J. E., \& Pólya, G. (1952). Inequalities (p. 89). Cambridge University Press.

Hennig, C., \& Kutlukaya, M. (2007). Some thoughts about the design of loss functions. REVSTATStatistical Journal, 5(1), 19-39.

Herden, G. (1989). On the existence of utility functions. Mathematical Social Sciences, 17(3), $297-313$.

Herden, G., \& Levin, V. L. (2012). Utility representation theorems for Debreu separable preorders. Journal of Mathematical Economics, 48(3), 148-154.

Jahn, J. (2009). Vector optimization. Springer.

Jaynes, E. T. (2003). Probability theory: The logic of science. Cambridge University Press.

Kaminski, B. (2007). On quasi-orderings and multi-objective functions. European Journal of Operational Research, 177(3), 1591-1598.

Li, Y., \& Busch, P. (2013). Von Neumann entropy and majorization. Journal of Mathematical Analysis and Applications, 408(1), 384-393.

Lieb, E. H., \& Yngvason, J. (1999). The physics and mathematics of the second law of thermodynamics. Physics Reports, 310(1), 1-96.

Lorenz, M. O. (1905). Methods of measuring the concentration of wealth. Publications of the American statistical association, 9(70), 209-219.

Maccheroni, F., Marinacci, M., \& Rustichini, A. (2006). Ambiguity aversion, robustness, and the variational representation of preferences. Econometrica, 74(6), 1447-1498.

Marshall, A. W., Olkin, I., \& Arnold, B. C. (1979). Inequalities: Theory of majorization and its applications. Springer.

Mehta, G. (1977). Topological ordered spaces and utility functions. International Economic Review, 779-782.

Mehta, G. (1981). Recent developments in utility theory. University of Queensland, Department of Economics.

Mehta, G. (1986a). Existence of an order-preserving function on normally preordered spaces. Bulletin of the Australian Mathematical Society, 34(1), 141-147.

Mehta, G. (1986b). On a theorem of fleischer. Journal of the Australian Mathematical Society, 40(2), 261-266.

Mehta, G. (1988). Some general theorems on the existence of order-preserving functions. Mathematical Social Sciences, 15(2), 135-143.

Minguzzi, E. (2010). Time functions as utilities. Communications in Mathematical Physics, 298(3), 855-868.

Minguzzi, E. (2013). Normally preordered spaces and utilities. Order, 30(1), 137-150.

Müller, M. P., \& Pastena, M. (2016). A generalization of majorization that characterizes shannon entropy. IEEE Transactions on Information Theory, 62(4), 1711-1720. 
Nachbin, L. (1965). Topology and order. 4. van Nostrand.

Nielsen, M. A. (1999). Conditions for a class of entanglement transformations. Physical Review Letters, $83(2), 436$.

Ok, E. A., et al. (2002). Utility representation of an incomplete preference relation. Journal of Economic Theory, 104(2), 429-449.

Ortega, P. A., \& Braun, D. A. (2013). Thermodynamics as a theory of decision-making with informationprocessing costs. Proceedings of the Royal Society A: Mathematical, Physical and Engineering Sciences, 469(2153), 20120683.

Pedersen, G. K. (2012). Analysis now (Vol. 118). Springer Science \& Business Media.

Peleg, B. (1970). Utility functions for partially ordered topological spaces. Econometrica: Journal of the Econometric Society, 93-96.

Rébillé, Y. (2019). Continuous utility on connected separable topological spaces. Economic Theory Bulletin, 7(1), 147-153.

Rényi, A., et al. (1961). On measures of entropy and information. In: Proceedings of the Fourth Berkeley Symposium on Mathematical Statistics and Probability, Volume 1: Contributions to the Theory of Statistics. The Regents of the University of California.

Richter, M. K. (1966). Revealed preference theory. Econometrica: Journal of the Econometric Society, $635-645$.

Schur, I. (1923). Uber eine klasse von mittelbildungen mit anwendungen auf die determinantentheorie. Sitzungsberichte der Berliner Mathematischen Gesellschaft, 22(9-20), 51.

Shore, J., \& Johnson, R. (1980). Axiomatic derivation of the principle of maximum entropy and the principle of minimum cross-entropy. IEEE Transactions on Information Theory, 26(1), 26-37. https://doi.org/10.1109/TIT.1980.1056144.

Still, S. (2009). Information-theoretic approach to interactive learning. EPL (Europhysics Letters), 85(2), 28005

Szpilrajn, E. (1930). Sur l'extension de l'ordre partiel. Fundamenta mathematicae, 1(16), 386-389.

Tishby, N., \& Polani, D. (2011). Information theory of decisions and actions. In Perception-action cycle (pp. 601-636). Springer.

Tsallis, C. (1988). Possible generalization of Boltzmann-Gibbs statistics. Journal of Statistical Physics, 52(1), 479-487.

Turgut, S. (2007). Catalytic transformations for bipartite pure states. Journal of Physics A: Mathematical and Theoretical, 40(40), 12185.

White, D. (1980). Notes in decision theory: Optimality and efficiency ii. European Journal of Operational Research.

Williams, R. J., \& Peng, J. (1991). Function optimization using connectionist reinforcement learning algorithms. Connection Science, 3(3), 241-268.

Publisher's Note Springer Nature remains neutral with regard to jurisdictional claims in published maps and institutional affiliations. 